\title{
Does the huamn mnid raed wrods as a wlohe?
}

\author{
Jonathan Grainger ${ }^{1}$ and Carol Whitney ${ }^{2}$ \\ ${ }^{1}$ LPC-CNRS, University of Provence, 13621 Aix-en-Provence, France \\ ${ }^{2}$ Department of Computer Science, University of Maryland, College Park, MD 20742, USA
}

A recent email message about a purported experiment run at Cambridge University provides a useful illustration of some fundamental mechanisms involved in reading. The message demonstrates that a text composed of words whose inner letters have been re-arranged can be raed wtih qutie anazimg esae! Although some of the readability of this email message is probably due to top-down factors made possible by the fact that almost $50 \%$ of the words are not mixed up, we suggest that a significant part of this 'jumbled word effect' is due to the special way in which the human brain encodes the positions of letters in printed words. Recent research using the masked-priming technique has helped to elucidate the mechanisms involved in letter-position coding. Masked primes are briefly presented, pattern-masked letter strings, whose effects on target processing are thought to reflect fast, automatic processing $[1,2]$. We will briefly describe two phenomena, relative-position priming and transposition priming, that have been observed with this paradigm and that are particularly relevant for understanding letter-position coding.

Masked-priming studies [1,2] have shown that targetword recognition is facilitated when primes are composed of a subset of the target word's letters (even when the proportion of shared letters is quite low, and absolute, length-dependent, letter position is violated), as long as the shared letters are in the same order in prime and target stimuli. That is, priming occurs only when relative positions are respected. For example, a six-letter word such as 'garden' is identified more rapidly when preceded by the masked prime 'grdn' compared to the unrelated condition 'pmts', and partly changing the order of letters (gdrn, nrdg) destroys the priming effect. This result would appear to be at odds with the apparent ease with which we can read the jumbled-word email message.

However, when primes share all their letters with target words, priming is robust with small changes in letter order, a phenomenon referred to as transposition priming. Thus, primes formed by transposing two adjacent letters in a target word (e.g. gadren-garden) facilitate word recognition compared with appropriate control primes $[3,4]$.

Most importantly, these two phenomena have propelled a new approach to letter-position coding that we believe provides a coherent account of the 'jumbled word effect'. We note in passing that current computational models of visual word recognition cannot capture these two key

Corresponding authors: Jonathan Grainger (grainger@up.univ-mrs.fr), Carol Whitney (cwhitney@cs.umd.edu). phenomena. For example, the well-known Interactive Activation [5] and Dual-Route Cascaded [6] models use a position-specific slot encoding, which is inconsistent with these experimental results. In response to the obvious shortcoming of these approaches, Whitney [7,8] proposed an encoding based on ordered letter pairs (e.g. the input 'take' is represented by activation of units representing TA, TK, TE, AK, AE, and KE). These units do not contain precise information about letter position, or about which letter is next to which. The same units (dubbed 'open bigrams') were later endorsed by Grainger and colleagues [4,9]. Open bigrams provide a convenient computational mechanism for representing the relative position of letters in a string. They are directly motivated by the relativeposition priming results $[1,2]$, but also provide a quite natural explanation for effects of transposed letters [3,4], and other key data (discussed by Whitney in $[7,8]$ ).

According to this open-bigram approach to letterposition coding, perception of printed words is relatively insensitive to letter transpositions because enough correct relative-position information is present in transposed stimuli. Thus, in the transposition priming experiments mentioned above, the prime 'gadren' has 92\% (11/12) overlap with the target 'garden', compared with $25 \%$ overlap (3/12) for the orthographic control prime 'galten' (note that in these calculations open bigrams are limited to a maximum of two intervening letters). According to classic accounts of letter-position coding, these two prime conditions do not differ in terms of their orthographic overlap with targets.

The superiority of this approach for explaining the 'jumbled word effect' can be illustrated with an example taken from the original email message. The jumbled version of the word 'only' ('olny') has 50\% orthographic overlap according to the coding schemes of the Interactive Activation and Dual-Route Cascaded models (and the same overlap with 'ogry', for example). Traditional bigram or trigram coding schemes do even worse with $20 \%$ overlap for bigrams (when spaces are included), and zero overlap for trigrams. On the other hand, the open-bigram scheme calculates overlap at $83 \%$ for this specific example, and is generally quite high for the complete set of words in the email message. Values range as a function of word length and the degree of transformation, for example with $80 \%$ overlap for 'porbelm' (problem), 67\% for 'wlohe' (whole), and a $53 \%$ minimum for 'bcuseae' (because). This relatively high level of orthographic overlap between the transformed word and the original word guarantees a minimum amount of correct bottom-up input for word identification. 


\section{References}

1 Humphreys, G.W. et al. (1990) Orthographic processing in visual word identification. Cogn. Psychol. 22, 517-560

2 Peressotti, F. and Grainger, J. (1999) The role of letter identity and letter position in orthographic priming. Percept. Psychophys. 61, 691-706

3 Perea, M. and Lupker, S.J. (2003) Transposed-letter confusability effects in masked form priming. In Masked Priming: State of the Art (Kinoshita, S. and Lupker, S.J., eds), pp. 97-120, Psychology Press

4 Schoonbaert, S. and Grainger, J. (in press) Letter position coding in printed word perception: effects of repeated and transposed letters. Lang. Cogn. Process.

5 McClelland, J.L. and Rumelhart, D.E. (1981) An interactive activation model of context effects in letter perception: Part 1 . An account of basic findings. Psychol. Rev. 88, 375-407
6 Coltheart, M et al. (2001) DRC: a dual-route cascaded model of visual word recognition and reading aloud. Psychol. Rev. 108, 204-256

7 Whitney, C. and Berndt, R.S. (1999) A new model of letter string encoding: simulating right neglect dyslexia. Prog. Brain Res. 121 $143-163$

8 Whitney, C. (2001) How the brain encodes the order of letters in a printed word: the SERIOL model and selective literature review. Psychonomic Bull. Rev. 8, 221-243

9 Grainger, J. and van Heuven, W.J.B. (in press) Modeling letter position coding in printed word perception. In The Mental Lexicon (Bonin, P., ed.), Nova Science, New York

1364-6613/\$ - see front matter $(1) 2003$ Elsevier Ltd. All rights reserved. doi:10.1016/j.tics.2003.11.006

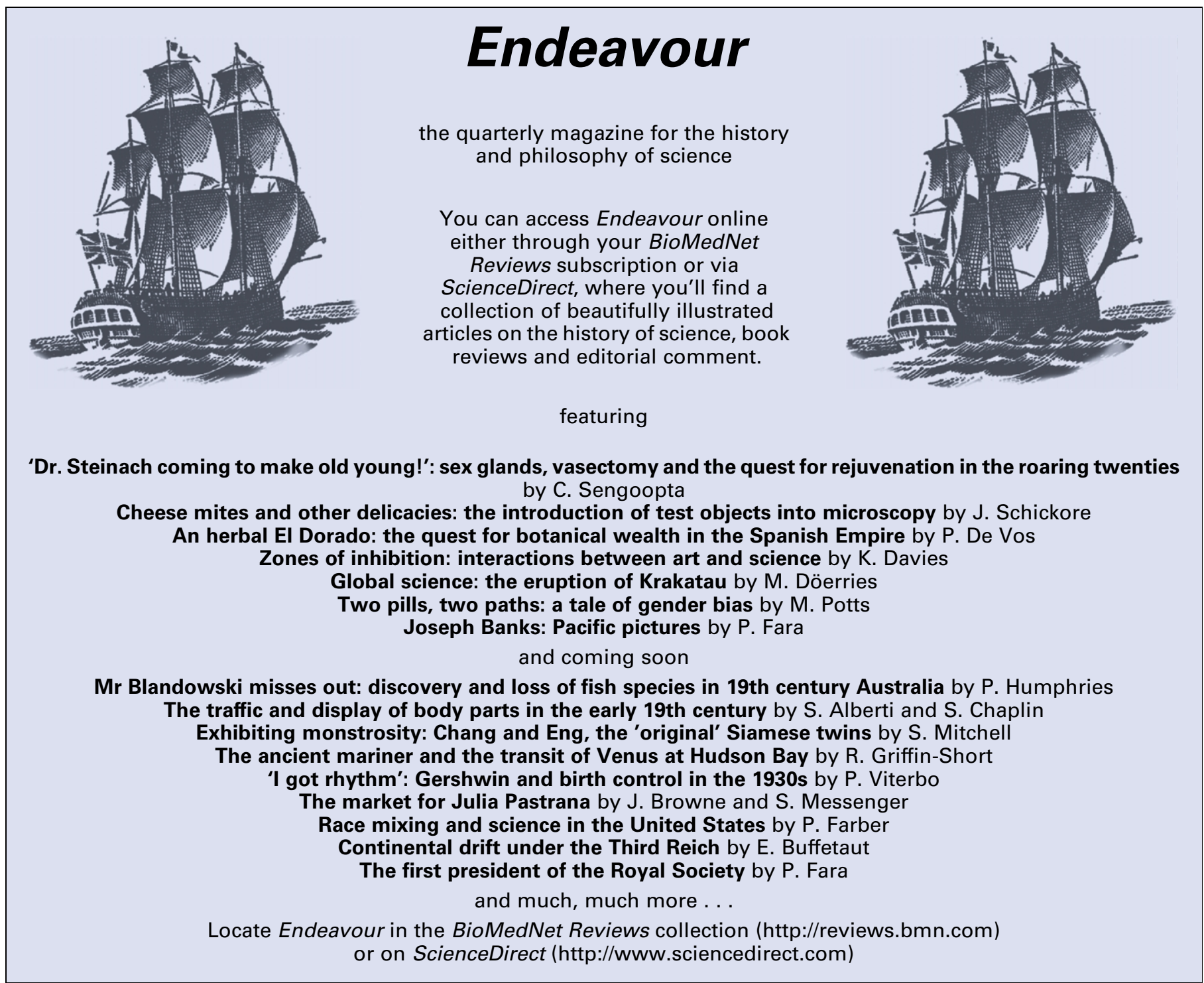

